\title{
Summary of the Electroweak and Beyond the Standard Model Working Group
}

\author{
J. Ferrando ${ }^{1}$, T. Nunnemann ${ }^{2}$, M. Spira ${ }^{3}$, M. Wessels ${ }^{4}$ \\ 1- Department of Physics, University of Oxford \\ Keble Road, Oxford, OX1 3RH, UK. \\ 2- Department für Physik, Ludwig-Maximillians Universität München \\ Am Coulomwall 1, D-85748 Garching, Germany. \\ 3- Paul Scherrer Institut \\ CH-5232 Villigen, Switzerland. \\ 4- Deutsches Elektronen-Synchrotron DESY \\ Notkestr. 85, D-22607 Hamburg, Germany.
}

\begin{abstract}
We present an overview of the Electroweak and Beyond the Standard Model Physics working group at the DIS2007 conference.
\end{abstract}

\section{Introduction}

For more than a century, high energy collisions of particles have been the golden method of investigating the ultimate structure of matter. Along with precision studies of heavy meson decays, primarily at lower energy colliders, these experiments have led to consolidation of the Standard Model (SM) as the theory of particle physics at energy scales up to $\mathcal{O}\left(10^{2}\right) \mathrm{GeV}$. Recent results from high energy colliders HERA and the TeVatron, complemented by the extraordinary precision measurements from the $b$-factories, display no significant deviations from SM predictions.

\section{The status of the Standard Model}

\subsection{Electroweak physics}

\subsubsection{Weak boson measurements at the TeVatron}

The mass of the $W$ boson is an important parameter of the Standard Model. Precise knowledge of the $W$ mass in combination with knowledge of the top quark mass results in a significant constraint on the mass of the Higgs boson. CDF have recently performed a new measurement of the $W$ mass using Run II data [2]. The $W$ mass is measured in the $W \rightarrow e \nu$ and $W \rightarrow \mu \nu$ decay channels by performing a binned maximum-likelihood fit to various kinematic distributions: lepton and neutrino $p_{T}$ and transverse mass $\left(M_{T}\right)$. The fit regions used are $65<M_{T}<90 \mathrm{GeV}$ and $p_{T}>32 \mathrm{GeV}, \mathbb{E}_{T}<48 \mathrm{GeV}$. The combination of all six fits gives $M_{W}=80413 \pm 34$ (stat) \pm 34 (syst) MeV.

The width of the $W$ boson has also been extracted by modelling the transverse mass distribution over the range $50-200 \mathrm{GeV}$. The data in the region $50-90 \mathrm{GeV}$ is used for normalisation. A fit for the width in the high transverse mass region $90-200 \mathrm{GeV}$ is then performed. This tail region, separated from the bulk of the resolution effects that determine the shape of the transverse mass distribution close to $M_{W}$, is sensitive to the width of the Breit-Wigner line-shape. The final measurement is $\Gamma_{W}=2032 \pm 71 \mathrm{MeV}$. 
Both of these measurements are the most precise single direct determinations of their kind, the $M_{W}$ measurement is compared to other measurements in Fig. 1.

The $M_{W}$ and $\Gamma_{W}$ results were obtained using approx $200 \mathrm{pb}^{-1}$ and $350 \mathrm{pb}^{-1}$ data. With over $2 \mathrm{fb}^{-1}$ data already delivered in Run II, prospects for improvement are good. The additional data are likely to yield not only an improvement of the statistical errors but also smaller systematic uncertainty due to the possibility of improving the precision to which, for example, the momentum scale is determined.

Diboson production measurements at the TeVatron also provide fertile ground for electroweak (EW) studies. The diboson cross-sections are sensitive to triple and quartic electroweak gauge-boson vertices predicted by the non-Abelian structure of the EW theory. Hence measurements of diboson production at the TeVatron are an important test of this part of the SM and provide a sensitive probe to any low energy

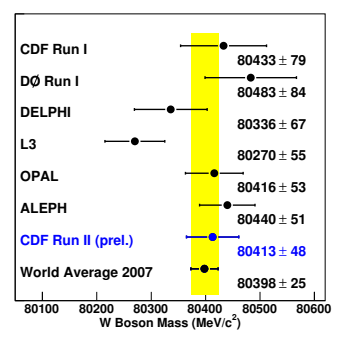

Figure 1: Summary of measurements of $M_{W}$ including the current world average. remnants of new physics at a higher scale. Various limits had been set on such processes in Run I, new studies of $W W, W Z, W \gamma, Z \gamma$ and $Z Z$ production have been performed by the $\mathrm{CDF}$ and D0 collaborations using Run II data [3].

The D0 collaboration has reported evidence for associated $W Z$ production, whilst the CDF collaboration were able to declare the first observation (at the $5.9 \sigma$ level) for the process. The CDF observation comes from $1.1 \mathrm{fb}^{-1}$ of Run II data, 16 data candidates are observed compared to an expected background of $2.7 \pm 0.28$ (stat) \pm 0.33 (syst) \pm 0.09 (lumi) events. The measured cross-section agrees well with the SM theoretical prediction.

Both D0 and CDF have performed measurements

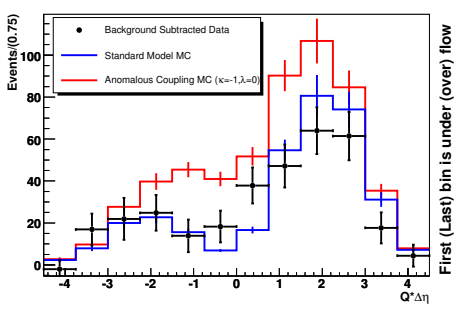

Figure 2: Comparison of the SM distribution and an anomalous $W W \gamma$ coupling distribution to the background subtracted charge-sign rapidity difference in the D0 $W \gamma$ analysis.

which would arise in the case of some new physics scenarios.

The CDF collaboration has reported the first evidence for $Z Z$ production from a search performed on $1.5 \mathrm{fb}^{-1}$ of Run II data. The search was performed using a combination of the four charged-lepton and two charged-lepton two neutrino final states. A $3.0 \sigma$ signal was extracted from a likelihood ratio fit. The measured cross-section $\sigma(p \bar{p} \rightarrow Z Z)=0.75_{-0.54}^{+0.71} \mathrm{pb}$ is consistent with the NLO calculation of the SM expectation. 


\subsubsection{Electroweak measurements at HERA}

The ZEUS collaboration has performed a measurement of Neutral Current (NC) ep Deep Inelastic Scattering (DIS) at HERA using polarised electron beams [5]. Figure 3 shows the asymmetry parameter $A^{ \pm} \propto a_{e} v_{q}$ where $a_{e}$ is the axial vector coupling to the electron and $v_{q}$ the vector coupling to the quark for this $e^{-} p$ data and previously published [6] ZEUS $e^{+} p$ data. The results are in good agreement with the SM and the asymmetry between the cross-section in left- and right-handed polarisations clearly shows the effect of parity violation as expected in the SM. In addition an H1-ZEUS working group has been set up to combine ZEUS and H1 data to produce the best possible precision HERA measurements of structure functions and electroweak parameters.

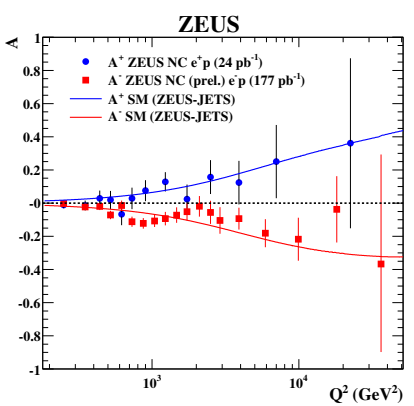

Figure 3: Measurement of the polarisation asymmetries $A^{ \pm}$by the ZEUS collaboration.

The H1 experiment, exploiting polarised electron and positron beams, performed a combined QCD and electroweak fit to their NC and Charged Current (CC) DIS data from the 1994-2005 running period [7]. This fit makes it possible to extract the electroweak $\mathrm{NC}$ vector $\left(v_{u}, v_{d}\right)$ and axial vector $\left(a_{u}, a_{d}\right)$ couplings of the $\mathrm{Z}$ boson to the light quarks. Figure 4 shows the H1 constraints in the $a_{u}-v_{u}$ plane. Results are in agreement with SM expectations and generally more precise than existing TeVatron constraints. Ambiguities from LEP constraints on the signs of the couplings are resolved.

\subsection{Top quark physics}

Until recently little was known about the top quark, what information we had came from statistically limited direct measurements at the TeVatron (Run I data) and indirect constraints from low energy data. Tops will be copiously produced at the LHC, but currently the only running accelerator with access to real top production is the TeVatron. With an order of magnitude more luminosity available in Run II than in Run I, it is now possible to perform top measurements that are no longer statistically limited. Many new measurements of top quark production and properties have recently been made $[8,9,10]$.

\subsubsection{Top production cross-section at the TeVatron}

At TeVatron top quarks are mainly produced in pairs via

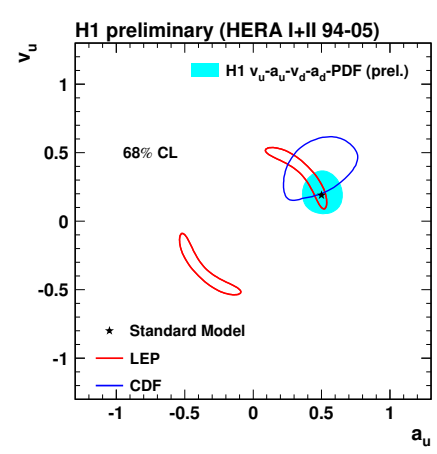

Figure 4: Results of the H1 combined QCD and Electroweak fit in the $a_{u}-v_{u}$ plane. the strong interaction, approximately $85 \%$ of the production cross-section arises from $q \bar{q}$ annihilation whilst the remaining $15 \%$ comes from gluon-gluon fusion. Theoretical calculations $[11,12]$ yield a total pair-production cross-section at TeVatron of order $7 \mathrm{pb}$. Higher cross-sections could be observed in the case of resonance or other non-SM production. The most recent measurements of the total pair production cross-section from CDF and D0 have made use of different $t \bar{t}$ decay channels in order to obtain independent results. 
The CDF collaboration has performed a measurement of the $t \bar{t}$-pair production crosssection in the dilepton final state using a lepton plus isolated track selection [8]. Although the choice of requiring an isolated track rather than a fully identified lepton candidate results in worse background, acceptance is significantly increased. Under the assumptions $M_{t}=175 \mathrm{GeV}$ and $\mathrm{BR}(W \rightarrow l \nu)=10.8 \%$ the measurement in this channel is translated to a measurement of the total cross-section of $\sigma(p \bar{p} \rightarrow t \bar{t})=9.0 \pm 1.3(\mathrm{stat}) \pm 0.5(\mathrm{sys}) \pm$ 0.5 (lumi) pb. This measurement is in good agreement with the SM prediction.

The D0 collaboration has recently performed measurements of $p \bar{p} \rightarrow t \bar{t}$ in lepton plus jets final states using a neural network $b$-tagging algorithm and in dilepton final states [8]. The cross-section measured in the dilepton channel $\sigma(p \bar{p} \rightarrow t \bar{t})=6.8_{-1.1}^{+1.2}(\mathrm{stat})_{-0.8}^{+0.9}(\mathrm{sys}) \pm$ 0.4 (lumi) pb is the most precise measurement in this decay channel. A higher precision is obtained in the $\ell+$ jets channel yielding $\sigma(p \bar{p} \rightarrow t \bar{t})=8.3_{-0.5}^{+0.6}$ (stat $)_{-1.0}^{+0.9}$ (sys) \pm 0.5 (lumi) pb. The precision of the CDF and D0 measurements is now approaching that of the theoretical predictions.

Top quarks may also be singly produced via electroweak processes at the TeVatron. The predicted NLO SM cross-section in the $s$-channel is $\sigma(p \bar{p} \rightarrow t b+X)=0.88 \pm 0.11 \mathrm{pb}$ and in the $t$-channel $\sigma(p \bar{p} \rightarrow t q b+X)=1.98 \pm 0.25 \mathrm{pb}$ [13]. More recent calculations including higher order soft gluon corrections are discussed in section 4.1. Single top quark events can be used to study the $W t b$ coupling [14] and to measure the magnitude of the quark-mixing matrix $[15,16]$ without assuming only three generations of quarks [17]. Although the single top production cross-section is of a similar order to the top pair production cross-section, the signal suffers from larger backgrounds (from $W+$ jet, $t \bar{t}$ and QCD multi-jet production). As a result of this, sophisticated analysis techniques must be employed to extract a signal.

The D0 collaboration has recently reported evidence for single top production at the $3.4 \sigma$ level [10]. Three different multivariate techniques were applied to $0.9 \mathrm{fb}^{-1}$ of Run II data. The techniques used were 'boosted' decision trees, the 'matrixelement' method and Bayesian neural networks. The cross-section itself was extracted using a Bayesian approach based on a binned likelihood over all bins and channels of the discriminant variable from each multivariate technique, separately for $t b+t q b, t q b$ and $t b$ analyses. All three multivariate techniques yield a non-zero crosssection, however the decision tree measurement is chosen for the main result since it is able to rule out the background-only hypothesis with greatest significance. The ex-

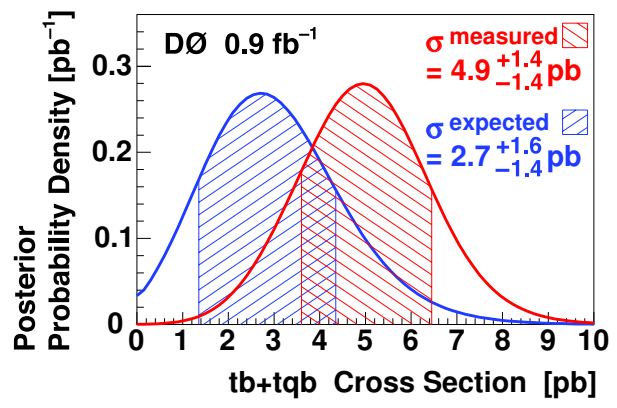

Figure 5: Expected SM and measured Bayesian posterior probability densities for the $t b+t q b$ cross-section obtained by the D0 collaboration. The shaded regions indicate one standard deviation above and below the peak positions. pected SM and measured posterior proba-

bilities for $t b+t q b$ are shown in Figure 5. The total measured cross-section is $\sigma(p \bar{p} \rightarrow$ $t b+X, t q b+X)=4.9 \pm 1.4 \mathrm{pb}$. This measurement is used to extract $0.68<\left|V_{t b}\right| \leq 1$ at $95 \%$ confidence level, without assuming CKM matrix unitarity.

The CDF collaboration has also performed a search for single top production using three different multivariate techniques. The techniques used by CDF were likelihood discrimi- 
nants, Bayesian neural networks and the 'matrix element' method. The largest signal that can be extracted comes from the matrix element technique and corresponds to a significance of $2.6 \sigma$. Figure 6 shows constraints on the Higgs arising from the most recent $M_{t}$ and $M_{W}$ measurements from TeVatron and results from LEP and SLD.

\subsubsection{Top quark properties}

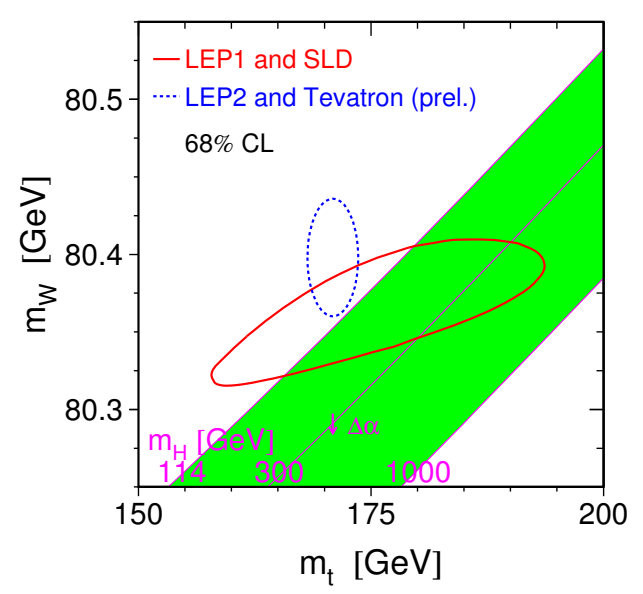

Figure 6: Constraints on the Higgs from measurements of $M_{W}$ and $M_{t}$.

hadronic $t \bar{t}$ decay channel results and dilepton channel results is $M_{t}=170.9 \pm 1.8 \mathrm{GeV}$.

The top quark charge has not yet been directly determined. One possible scenario is that the discovered top quark is an exotic quark of charge $4 e / 3$ while the top quark with charge $2 e / 3$ has mass $270 \mathrm{GeV}$ and has not yet been observed (XM) [18]. The CDF and D0 collaborations have both attempted to measure the charge of tops in $t \bar{t}$ production using $t \rightarrow b W,(W \rightarrow l \nu)$ decays. In this case the charge of the $W$-decay lepton is measured directly and the charge of the $b$-jet is measured using the momentum-weighted sum of the charges of the tracks in the jet. The charge of the lepton and jet are multiplied together and this distribution is compared to predictions from the SM and the XM. Using differing statistical methods both D0 and CDF obtain results strongly favouring the SM.

Measurements have also been made of the $W$-helicity in top decays and of the top branching fraction $R=\operatorname{Br}(t \rightarrow b W) / \operatorname{Br}(t \rightarrow q W)$ [9]. In both cases measurements from $\mathrm{CDF}$ and D0 are consistent with the SM prediction.

\subsection{Standard Model Higgs searches}

The experimental search for the SM Higgs boson has been ongoing since the 1970s. Direct limits from LEP suggest that the Higgs mass $M_{H}$ is greater than $114.4 \mathrm{GeV}$ at $95 \%$ confi- 
dence level. Indirect measurements from EW fits to measurements of $M_{t}$ and $M_{W}$ suggest $M_{H}=76_{-24}^{+33} \mathrm{GeV}$. Combining the LEP direct search data with the EW fits derive an upper limit of $M_{H}<182 \mathrm{GeV}$. New searches for the Higgs have been performed at the TeVatron [19].

The dominant decay modes for the SM Higgs boson are $H \rightarrow b \bar{b}$ for $M_{H}<135 \mathrm{GeV}$ and $H \rightarrow W W^{(*)}$ for $M_{H}>135 \mathrm{GeV}$. This leads to a twofold search strategy at the TeVatron. For $M_{H}<135 \mathrm{GeV}$ associated $W H$ and $Z H$ production with subsequent $H \rightarrow b \bar{b}$ are the processes of choice. For $M_{H}>135 \mathrm{GeV}, g g \rightarrow H$ production with subsequent $W W^{(*)}$ decay is the process searched for. The cross-section for $Z H(W H)$ production at the TeVatron is expected to be between $0.1(0.2) \mathrm{pb}$ and $0.01(0.03) \mathrm{pb}$, while for $g g \rightarrow H$ it is expected to be between 0.8 and $0.2 \mathrm{pb}$ depending on the Higgs mass. In all channels discovery of the Higgs remains immensely challenging; a considerable amount of effort has been invested in making sure to cover as many Higgs decay channels as possible over a wide phase space. New triggers and trigger algorithms have been developed by D0 and CDF, a large amount of effort has been devoted to improving $b$-tagging in order to reduce backgrounds, and jet energy corrections have been significantly improved yielding final results that have improved by more than just the factor expected by scaling luminosity.

A variety of analyses have been applied to CDF and D0 data [19]. Cut-based analyses have been employed as well as more sophisticated matrix-element- and neuralnetwork-based searches. No evidence for Higgs production has been observed and limits on the production cross-section compared to the SM have been set. The best individual limits have come from $H \rightarrow$ $W W \rightarrow l \nu l \nu$ searches where the CDF and D0 limits extend down to $3-4$ times the SM prediction. The combined D0 limits are presented in Figure 7, showing that the strongest limits are set on Higgs masses around $160 \mathrm{GeV}$. With about $8 \mathrm{fb}^{-1}$ of data expected to be taken by each experiment by

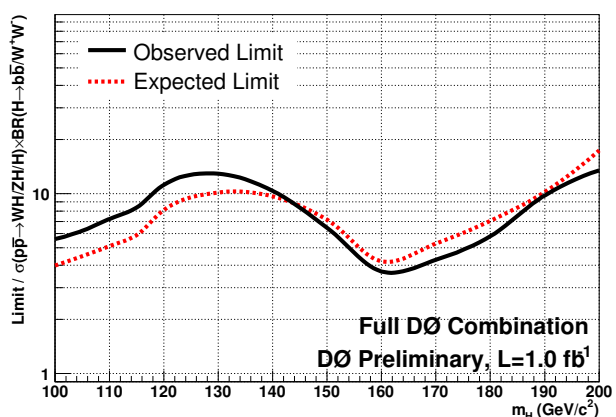

Figure 7: The D0 combined observed and expected cross-section limits on Higgs production, normalised to the SM prediction. the end of TeVatron running, improved statistical precision alone may not be sufficient to discover the Higgs, however considerable work is still being done to improve the reach of the TeVatron experiments. Improvements currently being studied include increasing lepton acceptance, further improving jet resolution and $b$-tagging as well as making more use of advanced analysis techniques such as those used in the TeVatron single top production searches.

\subsection{Flavour physics at the B-Factories}

The B-factories at SLAC and KEK continue to perform strongly accumulating very high statistics. Over 384 million $B B$ events have been collected by the Babar experiment at SLAC and over 535 million BB events by the Belle experiment at KEK. Both experiments have made significant progress in studies of $\mathrm{CP}$ violation and rare decays. 


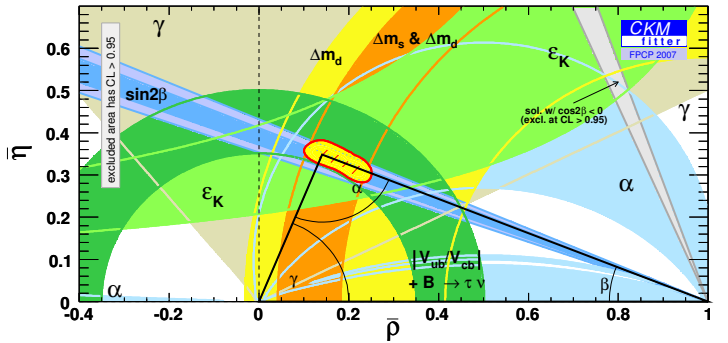

Figure 8: Constraints in the $\rho-\eta$ plane from recent measurements at B-factories

useful constraint on $\gamma\left(\phi_{3}\right)$.

Both Babar and Belle have studied a large number of rare B-decays as well as lepton flavour violating and rare decays of the $\tau$ lepton [22], observing no deviations from the SM and setting strong limits on new physics. Studies of $\tau \rightarrow \pi^{-} \pi^{0} \nu_{\tau}$ indicate a difference between the spectral function of the $\pi \pi$ system arising from tau decay compared to that arising from $e^{+} e^{-} \rightarrow \pi \pi$. This is very interesting in the context of $\frac{g_{\mu}-2}{2}$ measurements where it is expected to be possible to evaluate the dominant part of hadronic vacuum polarisation term in the theoretical calculation from the $\pi \pi$ spectral function in either $e^{+} e^{-}$or $\tau$ data.

On the theoretical side a new development has emerged by linking $B$ and $K$ physics calculations with high-energy collider data. Particular examples are strong constraints on $Z$-penguin dominated flavour-changing $K$ and $B$ decays, since the generic coupling $Z d^{i} \bar{d}^{j}$ of the $Z$ boson to any kind of down-type quarks is related to the $Z b \bar{b}$ coupling. The latter mediates the $Z \rightarrow b \bar{b}$ decay so that severe limits can be derived from the LEP and SLC data leading to significant suppressions of $Z$-penguin contributions [23].

\section{Beyond the Standard Model}

\subsection{Model-independent searches}

\subsubsection{Events with isolated leptons and missing transverse momentum at HERA}

Events with isolated leptons (electrons, muons and taus) and missing transverse momentum were observed in the HERA I data (1994-2000) [24, 25, 26, 27]. The only SM process which yields those events in ep collisions at HERA is single production of $W$ bosons. Events with large $P_{T}^{X}$, the transverse momentum of the hadronic final state, are of particular interest. Although no significant deviation from the SM was found, some atypical events with prominent jets have been observed in the electron and muon channels by the $\mathrm{H} 1$ collaboration and in the tau channel by the ZEUS collaboration.

The H1 search for isolated leptons has been updated to include data from the whole HERA I+II high-energy running 1994-2007 [28]. The overall yield of isolated leptons is in agreement with the SM predictions, however at large values of $P_{T}^{X}>25 \mathrm{GeV}$, the region sensitive to new physics, 21 electron or muon candidate events are observed in $e^{+} p$ data where $8.9 \pm 1.5$ events are expected from the SM. No excess is observed in $e^{-} p$ data. In the case of isolated tau leptons good agreement with the SM expectation is observed in all data sets, however the sensitivity to new physics is less than in the muon and electron channel due to the lower efficiency and purity. 

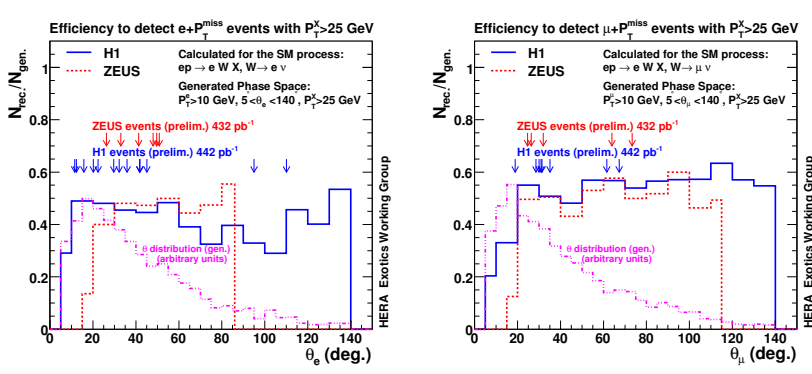

Figure 9: The efficiency of the H1 and ZEUS isolated lepton searches for $e p \rightarrow e W X$ (left $W \rightarrow e \nu_{e}$, right $\left.W \rightarrow \mu \nu_{\mu}\right)$ events with $P_{T}^{X}>25 \mathrm{GeV}$ as a function of the lepton polar angle $\left(\theta_{e, \mu}\right)$. The arrows in the figures denote the $\mathrm{H} 1$ and ZEUS candidate events. The generated spectrum for $e p \rightarrow e W X$ Monte Carlo is also shown.

H1 and ZEUS searches as a function of $P_{T}^{X}$ and as a function of the lepton polar angle $\theta_{l}$ have been made. The comparisons of the efficiencies of the two searches as a function of $P_{T}^{X}$ show that the ZEUS and H1 have similar efficiency for selecting $e^{ \pm} p \rightarrow e^{ \pm} W X$ events at high values of $P_{T}^{X}$. The more restricted phase space of the ZEUS search leads to a slightly lower efficiency than the H1 search. Figure 9 shows that in regions of the lepton polar angle $\theta_{e, \mu}$ common to both analyses the efficiencies of the two searches are similar. The majority of the $\mathrm{H} 1$ candidates lie within the ZEUS search acceptance, indicating that the ZEUS search is also sensitive to physics which could be responsible for the $\mathrm{H} 1$ excess, such as $\mathcal{R}$-Parity violating supersymmetry [30].

\subsubsection{Multi-lepton production at HERA}

The main multi-lepton production mechanism at HERA is photon-photon collision. H1 has performed a search for high transverse momentum ee, eee, $\mu \mu$ and $e \mu \mu$ topologies on the full HERA I+II set of $456 \mathrm{pb}^{-1} e^{ \pm} p$ data [31]. The invariant mass and summed scalar transverse momentum distributions $\left(\Sigma P_{T}\right)$, sensitive to new physics, for these classes of events are observed to be overall in agreement with the SM. In the $e^{+} p$ data only, four interesting events are observed with $\Sigma P_{T}>100 \mathrm{GeV}$.

The ZEUS collaboration has performed a search for ee and eee topologies on $446 \mathrm{pb}^{-1}$ $e^{ \pm} p$ HERA I+II data [32]. The ZEUS search suffers from a higher background, mainly arising from QED Compton events, than the $\mathrm{H} 1$ search. In both the ee and eee channels the ZEUS data are in agreement with the SM expectation.

\subsubsection{General BSM search at HERA}

A model-independent search for deviations from the SM was previously performed by H1 [33], using HERA I data. This search has been updated to the full HERA II data set [34], corresponding to $178 \mathrm{pb}^{-1}\left(159 \mathrm{pb}^{-1}\right) e^{+} p\left(e^{-} p\right)$ data. High $P_{T}$ final state configurations involving electrons $(e)$, muons $(\mu)$, jets $(j)$, photons $(\gamma)$ or neutrinos $(\nu)$ are considered. 
All final state configurations containing at least two such objects with $P_{T}>20 \mathrm{GeV}$ in the central region of the detector are investigated and classified into exclusive event classes.

The yields for the different classes for

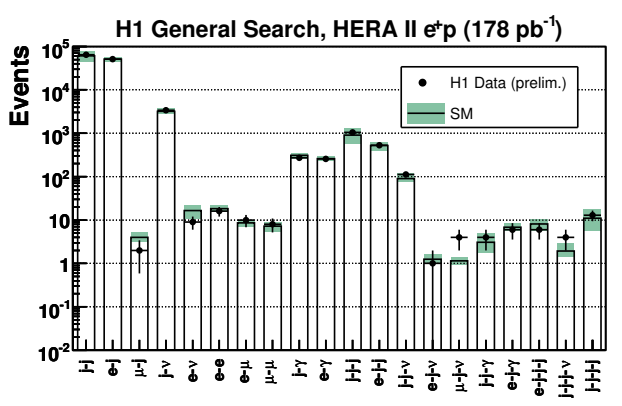
the $e^{+} p$ data are shown in Figure 10. Data events are found in 21 event classes each for $e^{+} p$ and $e^{-} p$ data and good agreement is observed between data and the SM expectations in most event classes. The largest disagreement in yield is in the $\mu-j-\nu$ topology for the $e^{+} p$ data, corresponding to the excess discussed in section 3.1.1. A non-biased statistical method is used to search for deviations of the data with respect to the SM in the summed scalar transverse momentum

Figure 10: The yields for the different event classes in the $\mathrm{H} 1$ general search, on $e^{+} p$, data compared to the SM predictions. $\left(\sum P_{T}\right)$ and total invariant mass $\left(M_{\text {all }}\right)$ distributions, sensitive to new physics, and to quantify their significance. Good agreement is observed in all event classes.

The data from the $\mathrm{H} 1$ general search has been added to QUAERO [35], providing a public interface to $\mathrm{H} 1$ and D0 data. With this interface, it is possible to quickly test any new physics models to data. The fact that the H1 general search covers all final states at HERA makes it ideal for this purpose. The performance of QUAERO on H1 and D0 data has been tested with example models such as leptoquark production and $\mathcal{R}$ parity violating SUSY. New data from H1 can now be easily added.

\subsubsection{Model-independent searches at the TeVatron}

Several model independent searches have been performed at the TeVatron [36]. The CDF collaboration has searched for $Z$ bosons with high $P_{T}$ in $0.94 \mathrm{fb}^{-1}$ of data. New physics models to which the search is sensitive include right-handed heavy quarks, technicolor particles, gauginos, squarks and excited states resulting from large extra dimensions. High $P_{T}$ $Z$ bosons are searched for inclusively and in $Z+X$ or $Z+Y+X$ final states, where $X$ and $Y$ can be leptons, photons, missing energy or large total transverse energy $\left(H_{T}\right)$. For each signature the observed $P_{T}(Z)$ spectrum and other distributions are compared with the SM expectations. No significant variation from the SM is observed.

The CDF collaboration has also searched for heavy objects decaying into high $P_{T}$ dileptons using $929 \mathrm{pb}^{-1}$ of Run II data. Acceptance is maximised by including all three generations of leptons, loosening fiducial requirements and using loose $P_{T}$ requirements. $b$-tagging is required in order to distinguish new physics involving the third generation quark. Dileptons are searched for in association with $X$, where $X$ may be large $H_{T}$, high $E_{T}$ jets, $b$ tags, third leptons or large missing $E_{T}$. The results show good agreement with the SM. To test the reach of the search a cross-section limit on a $300 \mathrm{GeV}$ right handed down type quark [37] is set using the electron and muon channels only. The limit is $1.4 \sigma_{Q}$ where $\sigma_{Q}=0.289 \mathrm{pb}$ is the expected SM cross-section. 


\subsection{SUSY searches}

Supersymmetry (SUSY) is a popular extension of the SM. SUSY associates supersymmetric partners (sparticles) with the known particles and unifies internal symmetries with Lorentz invariance. Supersymmetric models provide solutions to many problems of the SM, such as fine-tuning, unification and hierarchy, and predict spectacular final states in high-energy particle collisions. Despite extensive studies at colliders and elsewhere, no trace of SUSY has yet been found. Many new searches for signatures of SUSY have been performed at the TeVatron [38]. Two of the most popular scenarios for searches are the minimal supersymmetric extension of the SM (MSSM) and minimal Supergravity (mSUGRA).

MSSM is the simplest realistic SUSY theory. It requires two Higgs doublets resulting in a Higgs sector with two charged and three neutral scalar bosons. Assuming $C P$-invariance, one of the neutral bosons $(A)$ is $C P$-odd, and the other two $(h, H)$ are $C P$ even. The Yukawa couplings of $A$ to down-type particles (e.g. $\tau$ lepton or $b$ quark) are enhanced by a factor $\tan \beta$ relative to the SM. The leading decay modes of $A$ and the corresponding degenerate $C P$ even Higgs boson are $\phi \rightarrow b \bar{b}(\sim 90 \%)$ and $\phi \rightarrow \tau \tau$ $(\sim 10 \%)$. Both CDF and D0 have searched in the $\tau \tau$ decay channel, where the QCD background is lower. The results of the CDF search in the $\tau_{e} \tau_{\text {had }}$ and $\tau_{\mu} \tau_{\text {had }}$ channels combined are shown in Fig. 11. No evidence for $M_{A}=90-250 \mathrm{GeV}$ is observed, a small excess of events exists with a significance less than $2 \sigma$ when the entire mass range is considered. No excess above the expected backgrounds is observed in the D0 search. The two experiments have set limits within the framework of the MSSM for Higgs masses in the range 90 to $250 \mathrm{GeV}$.

Associated $\tilde{\chi}_{1}^{ \pm} \tilde{\chi}_{2}^{0}$ production can lead to clean multi-lepton final states when $p \bar{p} \rightarrow \tilde{\chi}_{1}^{ \pm} \tilde{\chi}_{2}^{0}$ is followed by $\tilde{\chi}_{2}^{0} \rightarrow l l \tilde{\chi}_{1}^{0}$ and $\tilde{\chi}_{1}^{ \pm} \rightarrow l \nu \tilde{\chi}_{1}^{0}$. The CDF (D0) collaboration has performed 14 (6) searches respectively for such final states each using up to $1.0(1.1) \mathrm{fb}^{-1}$ of Run II data. No evidence for SUSY is observed and

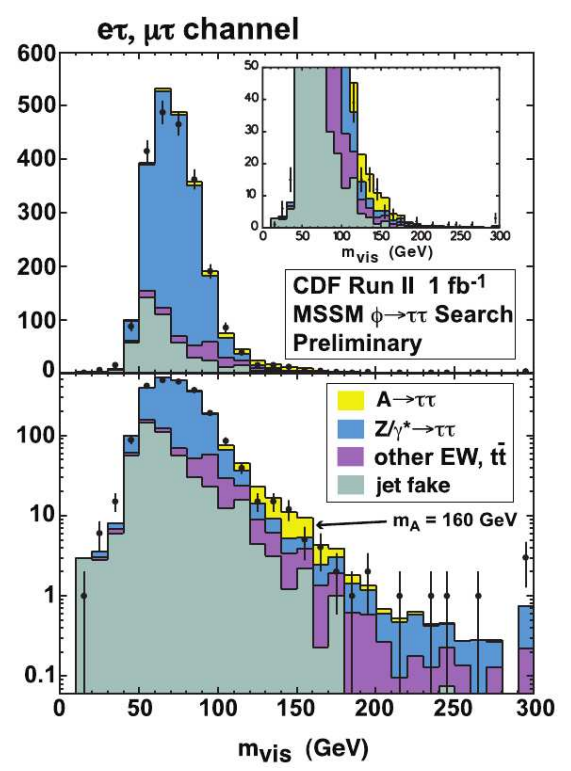

Figure 11: Partially reconstructed di-tau mass in the $A \rightarrow \tau \tau \mathrm{CDF}$ search. The normalisation of backgrounds and signal $\left(M_{A}=160 \mathrm{GeV}\right)$ correspond to fit results for the signal exclusion. limits have been set on the production cross-section multiplied by the branching ratio to three leptons of $0.2(0.08) \mathrm{pb}$ for $M(\tilde{\chi})=140 \mathrm{GeV}$, compared to expected limits of the order of $0.1 \mathrm{pb}$.

The D0 collaboration has also updated their search for scalar quark, $\tilde{q}$, and gluino, $\tilde{g}$, production using their $1 \mathrm{fb}^{-1}$ data set. An exclusion region as function of $M_{\tilde{q}}$ and $M_{\tilde{g}}$ was derived, yielding lower mass limits of $M_{\tilde{q}}>375 \mathrm{GeV}$ and $M_{\tilde{g}}>289 \mathrm{GeV}$, respectively.

\subsection{Leptoquarks}

Leptoquarks (LQs) are hypothetical bosons which couple to a lepton and a quark via a Yukawa coupling (denoted $\lambda$ ). In the SM, both quarks and leptons occur in left-handed 
$S U(2)$ doublets and right-handed $S U(2)$ singlets. The symmetry between quarks and leptons leads to the cancellation of triangle anomalies which make the SM renormalizable. Leptoquarks appear in theories in which this symmetry is more fundamental.

Leptoquarks are colour triplets which would be pair-produced in either $q \bar{q}$ or $g g$ interactions at $p \bar{p}$ or $p p$ colliders. Since LQs carry electroweak charge, they would also be produced in $e^{+} e^{-}$collisions. Only SM gauge couplings are involved in pair-production of scalar LQs. Therefore the cross-sections depend neither on the quark-lepton-LQ Yukawa coupling nor on the quark and lepton generations to which the leptoquark couples. In contrast, leptoquarks would be singly produced via the Yukawa coupling in lepton-quark collisions. Searches at $e p$ colliders are sensitive only to LQs which couple to electrons and the sensitivity to LQs which couple to the second and third generation quarks is far below that of first-generation LQs. Leptoquarks are usually (but not always) assumed to be generation diagonal. Models in which LQs couple to more than one generation of quarks or leptons would induce flavour-changing neutral currents or lepton flavour violation (LFV), respectively.

LQs can be classified into 14 types with respect to the quantum numbers spin, isospin and chirality within the framework of the Buchmüller-Rückl-Wyler (BRW) model [39]. Leptoquarks carry both lepton (L) and baryon (B) quantum numbers. The fermion number $F=L+3 B$ is assumed to be conserved.

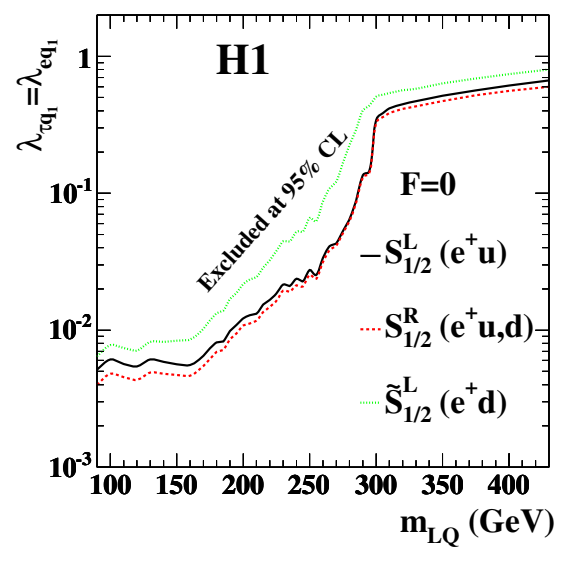

Figure 12: Limits on the coupling constants $\lambda_{\tau q_{1}}=\lambda_{e q_{1}}$ as a function of the LQ mass $M_{L Q}$ for $\mathrm{F}=0$ scalar LQs. Regions above the lines are excluded at $95 \% \mathrm{CL}$. The notation $q_{1}$ indicates that only processes involving first generation quarks are considered. of $F=2$ for $e^{-} q$ processes and $F=0$ for $e^{+} q$ processes. For $M_{L Q}$ well below the $e p$ centre-ofmass energy, the $s$ channel production dominates. For $M_{L Q}$ greater than the centre-of-mass energy the $s$ and $u$ channel processes become of equal importance. The BRW model assumes lepton flavour conservation such that the LQs produced in $e p$ collisions decay only to $e X$ or $\nu_{e} X$ final states. A general extension of the BRW model allows for the decay of LQs to final states containing a lepton of different flavour $(\mu$ or $\tau)$ and a jet. Non-zero couplings to 
an $e q$ pair and to a $\mu q$ or $\tau q$ pair are assumed.

In the $\mathrm{H} 1$ analysis, high $P_{T}$ muon and tau signatures are searched for and no evidence for LFV is found. Limits on couplings to 14 different LQs as a function of $M_{L Q}$ are derived. An example of the limits under the assumption that the tau- and electron-first generation quark couplings are equal is shown in Figure 12. The H1 results are directly comparable to previous limits set by ZEUS [44] and are found to be similar. Lower mass limits on the first and second generation leptoquarks from hadron-hadron collisions extend up to about $250 \mathrm{GeV}[45,46]$, lower mass bounds from $e^{+} e^{-}$annihilation reach values of $100 \mathrm{GeV}[47]$.

\subsection{Other non-SUSY BSM models}

The proliferation of fermions can naturally be explained if the SM fermions are composite, in which case excited states may exist. A minimal extension $[48,49,50]$ of the SM can incorporate excited fermions such as excited leptons $\left(l^{*}\right)$. Considering only EW gauge mediated interactions (GMI), the excitation part of the Lagrangian is:

$$
\mathcal{L}_{F^{*} F}=\frac{1}{2 \Lambda} \overline{F_{R}^{*}} \sigma^{\mu \nu}\left[g f \frac{\vec{\tau}}{2} \overrightarrow{W_{\mu \nu}}+g^{\prime} f^{\prime} \frac{Y}{2} B_{\mu \nu}\right] F_{L}+\text { h.c. },
$$

where the new weights $f$ and $f^{\prime}$ multiply the SM coupling constants $g$ and $g^{\prime}$ corresponding to the weak $S U(2)$ and electromagnetic $U(1)$ sectors respectively. The corresponding gauge boson fields are denoted by $W$ and $B$, the matrix $\sigma_{\mu \nu}=(i / 2)\left[\gamma^{\mu}, \gamma^{\nu}\right], \tau$ are the Pauli matrices and $Y$ is the hypercharge. The compositeness scale $\Lambda$ reflects the range of the new confinement force and, together with $f$ and $f^{\prime}$, determines the production cross-section.

The H1 collaboration has recently performed a search for excited leptons at HERA using $434 \mathrm{pb}^{-1} e^{ \pm} p$ data [51]. Both excited neutrinos $\left(\nu^{*}\right)$ and excited electrons $\left(e^{*}\right)$ have been searched for. Sensitivity to $\nu^{*} \mathrm{~s}$ is much higher in $e^{-} p$ collisions due to helicity enhancement specific to CC like processes and only the $e^{-} p$ data was used to search for $\nu^{*}$ s. The search was performed for the decay channels $\nu^{*} \rightarrow \nu \gamma, \nu^{*} \rightarrow \nu Z$ and $\nu^{*} \rightarrow W e$, using six subsequent decay channels, covering approximately $90 \%$ of the total branching fraction. No evidence for $\nu^{*} \mathrm{~s}$ is observed and limits on the crosssection are set and translated to limits in the $f / \Lambda-M_{\nu^{*}}$ plane, with $M_{\nu^{*}}$ the mass of the excited neutrino. The limits are set for

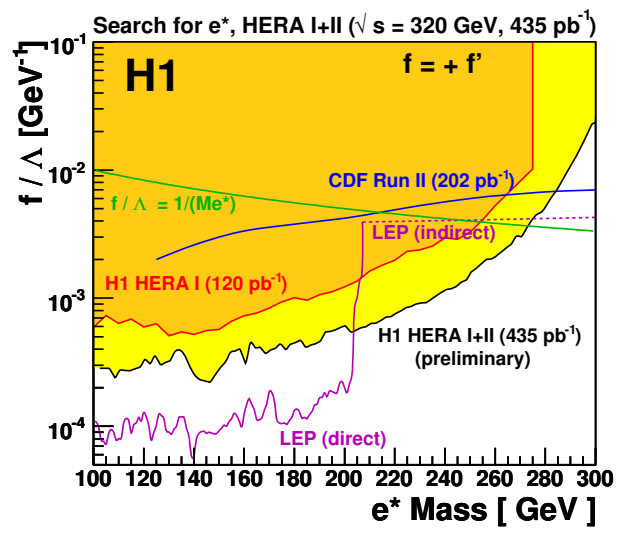
two scenarios: $f=f^{\prime}$ (no $\gamma \nu^{*} \nu$ coupling) and $f=-f^{\prime}$ (maximal $\gamma \nu^{*} \nu$ coupling). For $f=-f^{\prime}$ and $f / \Lambda=1 / M_{\nu^{*}}$, a lower limit of $M_{\nu^{*}}>211 \mathrm{GeV}$ is obtained. These limits explore new domains and significantly improve on previous LEP limits.

The full HERA I+II $434 \mathrm{pb}^{-1} e^{ \pm} p$ data set was used in the $e^{*}$ search. The decay channels $e^{*} \rightarrow e \gamma, e \rightarrow e Z$ and $e \rightarrow \nu W$ with subsequent hadronic decay of the weak bosons were studied. In the case of $f=-f^{\prime}$ (no $\gamma e^{*} e$ coupling) the production cross-section is 
suppressed, therefore only the maximal $\gamma e^{*} e$ coupling case $\left(f=f^{\prime}\right)$ was considered. No excess over the SM is observed in the data and limits are set in the $f / \Lambda-M_{e^{*}}$ plane. For $f / \Lambda=1 / M_{e^{*}}$ a lower bound of $M_{e^{*}}>273 \mathrm{GeV}$ is set. Figure 13 shows these results in comparison with limits obtained at the TeVatron assuming that the excited electrons are only produced via GMI.

At the TeVatron production of excited leptons is dominated by contact interactions rather than GMI. A search for $e^{*} \rightarrow e \gamma$ has been performed by the D0 collaboration [36] using $1.0 \mathrm{fb}^{-1}$ of Run II data. Limits have been set in the $\Lambda-M_{e^{*}}$ plane, yielding a lower mass limit of $M_{e^{*}}>756 \mathrm{GeV}$ for a compositeness scale of $\Lambda=1 \mathrm{TeV}$.

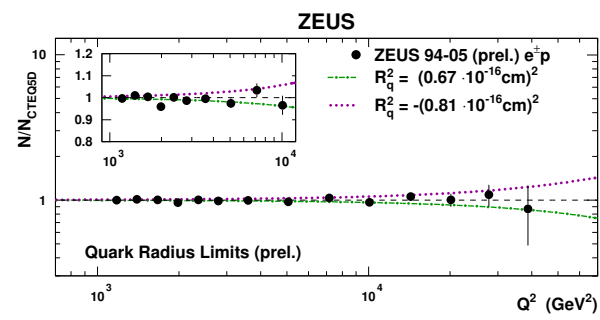

Figure 14: ZEUS limits on the quark radius obtained using the CI search. The points show the ratio of the data to the $\mathrm{SM}$ predictions in bins of $Q^{2}$. The expected deviation for the derived quark radius limits are also shown.

The ZEUS collaboration has exploited the precision of their high $Q^{2}$ NC DIS measurements to search for new physics within the framework of four-fermion contact interactions (CIs) [52]. The addition of CIs adds new terms to the NC SM Lagrangian. By comparing the deviations of the data from the SM predictions and parametrising possible effects using the CI formalism limits are set on contact interactions themselves and various other specific models, such as heavy leptoquarks, large extra dimensions and finite-radius quarks. An example of the limits expressed in terms of the quark radius is shown in Figure 14, an upper limit of $0.67 \times 10^{-4} \mathrm{fm}$ is set.

A host of other BSM searches too numerous to cover in detail here has been performed at the TeVatron [36]. Models such as fourth generation quarks, extra gauge bosons ( $W^{\prime}$ and $Z^{\prime}$ ) and extra dimensions have all been tested and no evidence for new physics has been found.

\section{A theoretical perspective}

\subsection{Single top production and top decay}

Recent calculations for single top production at the LHC and TeVatron [53] have applied NNNLO soft-gluon corrections, leading to corrections of the order of $3-3.5 \%$ relative to NNLO calculations. For all channels at TeVatron the cross-sections for single top and single anti-top production are identical, whereas at the LHC the cross-section in the $s$ and $t$ channels is larger for single top production than for anti-top production.

Single top production and top decay including spin correlations have been recently implemented in the event generator MC@NLO [54]. For this implementation the NLO single top production had to be computed using the FKS subtraction method [55] and MC counter terms had to be calculated. Studies of spin correlations in (single) top decay can determine the handedness of the electroweak coupling of the top. The top-spin is very strongly correlated to the decay lepton production making it possible to measure the coupling. Angular correlations in top decay were implemented in MC@NLO precise to NLO for real (hard) emissions and LO for soft or collinear emissions. The effect of the spin correlations on the 
lepton decay angle relative to the hardest jet which does not contain a stable $b$ hadron is shown in Figure 15.

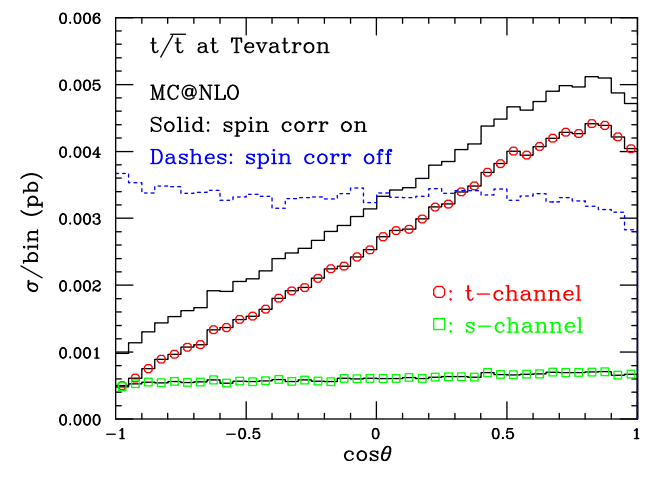

Figure 15: Effect of spin correlations on the lepton decay angle $\theta$ relative to the hardest jet which does not contain a stable $b$ hadron for single $t / \bar{t}$ production at the TeVatron.
Single top studies have also been preformed using the Monte Carlo code MCFM [56]. MCFM has been extended to include the three single top channels and correctly deal with the full spin correlation of the leptonic decay of the top. Soft and collinear divergences were treated according to the subtraction method of Catani, Dittmaier, Seymour and Trócsányi [57].

\section{$5 \quad$ Future colliders}

\subsection{LHC physics prospects}

With the LHC-era fast approaching, many studies have been performed on the physicsreach of the LHC experiments using the first two years of data [58]. It is reasonable to expect data sets of approximately $5 \mathrm{fb}^{-1}$ providing machine schedules are adhered to. Although the initial emphasis of LHC physics will be on understanding detector performance and SM processes there will be sensitivity to new physics. In the case of high mass di-lepton resonances, $Z^{\prime}\left(W^{\prime}\right)$, where the background is low the prospects of an early discovery are good for new physics in the $\mathrm{TeV}$ mass region. Both ATLAS and CMS could start to probe SUSY at $1-2 \mathrm{TeV}$ and there would be high potential for BSM physics discoveries such as Majorana neutrinos and technicolor. It is possible that the $H \rightarrow W W$ channel will allow evidence of a SM Higgs boson at $M_{H} \sim 165 \mathrm{GeV}$ with $1 \mathrm{fb}^{-1}$ luminosity, while the $H \rightarrow Z Z$ channel will allow discovery in the mass range $190-450 \mathrm{GeV}$ with $4 \mathrm{fb}^{-1}$. At least $10 \mathrm{fb}^{-1}$ would be required for a MSSM Higgs $5 \sigma$ discovery.

The sensitivity of the LHC searches are affected by the precision to which the proton parton distribution functions (PDFs) are known [59]. Uncertainties on the PDFs lead directly to uncertainties on both SM processes such as $W, Z, t$ and $H$ production and BSM processes such as $h H^{+}$and $a H^{+}$production in the MSSM. The new CTEQ6.5M PDF shifts the total cross-section, $\sigma_{\text {tot }}$ relative to CTEQ6.1M by similar magnitudes to the PDF uncertainties, cross-sections for specific new physics processes are also modified by up to $20 \%$. With this in mind it is clear that further precision measurements of the PDFs will be of significant value to searches at the LHC.

Electroweak and top quark measurements will be amongst the first studied by both ATLAS [60] and CMS [61]. Large $W$ and $Z$ boson samples should be accrued in early running offering valuable tools for the understanding of the performance of the detectors. With sufficiently precise PDFs the $W$ and $Z$ production processes could be used to check luminosity measurements. $t \bar{t}$ production will provide useful samples for jet energy scale and $b$-tagging efficiency. Early and basic $W, Z$ and top measurements could be made using $100 \mathrm{pb}^{-1}$ to $1 \mathrm{fb}^{-1}$ of data. Indeed with only $1 \mathrm{fb}^{-1}$ of data, CMS expect their precision to 
already be dominated by systematics in their measurements of $W$ and $Z$ production.

\subsection{ILC physics prospects}

A future high energy linear $e^{+} e^{-}$collider such as the ILC could complement results from the LHC [62]. If one considers a scenario where EW symmetry breaking is realised through the Higgs mechanism and where low energy SUSY exists, then the prospective measurements from the ILC would greatly enhance knowledge obtained from LHC measurements. In such a scenario the LHC experiments would likely discover the Higgs and explore the strongly-interacting SUSY sector (squarks and gluinos) and make measurements of moderate precision that many of which will be model-dependent. The ILC would then be able to make high precision measurements of the Higgs sector and a detailed exploration of the electroweakly interacting SUSY sector (sleptons and gauginos). In addition there would be possible discoveries in regions of parameter space to which the LHC would not be sensitive.

There are several examples of possible measurements at ILC which will not be possible at the LHC. The ILC can detect the Higgs boson independent of the decay mode even if it decays into invisible particles, using the recoil mass spectrum in $Z H \rightarrow(e e, \mu \mu) X$. The ILC can also establish the spin of the Higgs. The sensitivity to the electroweakly interacting SUSY sector with clean signatures and low backgrounds makes precise SUSY spectroscopy possible at the ILC. The physical observables within the LHC and ILC reach would make it possible to constrain the SUSY Lagrangian. With many other possible physics topics to be searched for at the ILC, it is clear that the ILC is likely to greatly increase our knowledge of the electroweak sector and possible BSM scenarios.

\section{Conclusion and outlook}

This is an exciting time for EW and BSM physics. With high energy HERA running finished the $\mathrm{H} 1$ and ZEUS collaborations are beginning to produce their final statements on many searches for new physics, so far the SM is holding up well. The polarised running of HERA II has been a success, resulting in increasingly precise constraints from the HERA experiments on EW parameters. The performance of the TeVatron in Run II has been excellent and this has been reflected in a clutch of impressive results. The high precision measurement of $M_{W}$ together with improving $M_{t}$ determination is reflected in stronger constraints on the SM Higgs boson mass. Direct searches for the SM Higgs boson an new physics are showing ever improving sensitivity. With an order of magnitude more data statistics expected by the end of Run II, TeVatron data could yet yield the discovery of the Higgs. We await the imminent start of the LHC-era. Though early work will focus on detector-commissioning and calibration using standard candles, it will be possible to search previously unexplored regions of phase-space for new physics with the first two years of LHC data.

\section{Acknowledgements}

We thank all participants in the parallel session for interesting talks and lively discussion.

\section{References}

[1] Slides:

http: //indico. cern. ch/contributionDisplay . py? contribId $=16 \&$ sessionId $=2 \&$ conf $I d=9499$ 
[2] CDF, S. Malik, These proceedings.

[3] CDF/D0, Y. Maravin, These proceedings.

[4] U. Baur, S. Errede and G. L. Landsberg, Phys. Rev. D50, 1917 (1994), [hep-ph/9402282].

[5] ZEUS, S. Bhadra, These proceedings.

[6] ZEUS, S. Chekanov et al., Phys. Lett. B637, 210 (2006), [hep-ex/0602026].

[7] H1, G. Li, These proceedings.

[8] CDF/D0, C. Gerber, These proceedings.

[9] CDF/D0, J. Wagner, These proceedings.

[10] CDF/D0, S. Jabeen, These proceedings.

[11] N. Kidonakis and R. Vogt, Phys. Rev. D68, 114014 (2003), [hep-ph/0308222].

[12] M. Cacciari, S. Frixione, M. L. Mangano, P. Nason and G. Ridolfi, JHEP 04, 068 (2004), [hep$\mathrm{ph} / 0303085]$.

[13] Z. Sullivan, Phys. Rev. D70, 114012 (2004), [hep-ph/0408049].

[14] A. P. Heinson, A. S. Belyaev and E. E. Boos, Phys. Rev. D56, 3114 (1997), [hep-ph/9612424].

[15] N. Cabibbo, Phys. Rev. Lett. 10, 531 (1963).

[16] M. Kobayashi and T. Maskawa, Prog. Theor. Phys. 49, 652 (1973).

[17] G. V. Jikia and S. R. Slabospitsky, Sov. J. Nucl. Phys. 55, 1387 (1992).

[18] D. Chang, W.-F. Chang and E. Ma, Phys. Rev. D59, 091503 (1999), [hep-ph/9810531].

[19] CDF/D0, R. Vilar, These proceedings.

[20] Babar, N. Barlow, These proceedings.

[21] Belle, I. Nakamura, These proceedings.

[22] Belle, H. Hayashii, These proceedings.

[23] U. Haisch, These proceedings.

[24] H1, V. Andreev et al., Phys. Lett. B561, 241 (2003), [hep-ex/0301030].

[25] ZEUS, S. Chekanov et al., Phys. Lett. B559, 153 (2003), [hep-ex/0302010].

[26] ZEUS, S. Chekanov et al., Phys. Lett. B583, 41 (2004), [hep-ex/0311028].

[27] H1, A. Aktas et al., Eur. Phys. J. C48, 699 (2006), [hep-ex/0604022].

[28] H1, Y. De Boer, These proceedings.

[29] ZEUS, K. Korcsak-Gorzo, These proceedings.

[30] S. Y. Choi et al., arXiv:hep-ph/0612302.

[31] H1, G. Brandt, These proceedings.

[32] ZEUS, O. Ota, These proceedings.

[33] H1, A. Aktas et al., Phys. Lett. B602, 14 (2004), [hep-ex/0408044].

[34] ZEUS, E. Sauvan, These proceedings.

[35] S. Caron, These proceedings.

[36] CDF/D0, D. Stuart, These proceedings.

[37] J. D. Bjorken, S. Pakvasa and S. F. Tuan, Phys. Rev. D66, 053008 (2002), [hep-ph/0206116].

[38] CDF/D0, R. Ströhmer, These proceedings.

[39] W. Buchmuller, R. Ruckl and D. Wyler, Phys. Lett. B191, 442 (1987).

[40] H1, A. Aktas et al., Phys. Lett. B629, 9 (2005), [hep-ex/0506044].

[41] ZEUS, S. Chekanov et al., Phys. Rev. D68, 052004 (2003), [hep-ex/0304008].

[42] H1, A. Dubak, These proceedings.

[43] H1, A. Aktas et al., hep-ex/0703004.

[44] ZEUS, S. Chekanov et al., Eur. Phys. J. C44, 463 (2005), [hep-ex/0501070].

[45] D0, V. M. Abazov et al., Phys. Lett. B636, 183 (2006), [hep-ex/0601047].

[46] CDF, A. Abulencia et al., Phys. Rev. D73, 051102 (2006), [hep-ex/0512055].

[47] OPAL, G. Abbiendi et al., Eur. Phys. J. C31, 281 (2003), [hep-ex/0305053].

[48] K. Hagiwara, D. Zeppenfeld and S. Komamiya, Z. Phys. C29, 115 (1985).

[49] U. Baur, M. Spira and P. M. Zerwas, Phys. Rev. D42, 815 (1990).

[50] F. Boudjema, A. Djouadi and J. L. Kneur, Z. Phys. C57, 425 (1993). 
[51] H1, N. Trinh, These proceedings.

[52] ZEUS, S. Schlenstedt, These proceedings.

[53] N. Kidonakis, These proceedings.

[54] P. Motylinski, These proceedings.

[55] S. Frixione, Z. Kunszt and A. Signer, Nucl. Phys. B467, 399 (1996), [hep-ph/9512328].

[56] F. Tramontano, These proceedings.

[57] S. Catani, S. Dittmaier, M. H. Seymour and Z. Trocsanyi, Nucl. Phys. B627, 189 (2002), [hep$\mathrm{ph} / 0201036]$.

[58] ATLAS/CMS, D. Rebuzzi, These proceedings.

[59] C. Yuan, These proceedings.

[60] ATLAS, A.-C. Le Bihan, These proceedings.

[61] CMS, F.-P. Schilling, These proceedings.

[62] ILC, A. Raspereza, These proceedings. 